\title{
Boundary regularity for polyharmonic maps in the critical dimension
}

\author{
Tobias Lamm and Changyou Wang \\ Communicated by F. Duzaar
}

\begin{abstract}
We consider the Dirichlet problem for intrinsic and extrinsic $k$-polyharmonic maps from a bounded, smooth domain $\Omega \subseteq \mathbb{R}^{2 k}$ to a compact, smooth Riemannian manifold $N \subseteq$ $\mathbb{R}^{l}$ without boundary. For any smooth boundary data, we show that any $k$-polyharmonic map $u \in W^{k, 2}(\Omega, N)$ is smooth near the boundary $\partial \Omega$.
\end{abstract}

Keywords. Polyharmonic maps, boundary regularity.

AMS classification. 58E20, 35J35, 35J40.

\section{Introduction}

For $k \in \mathbb{N}$ let $\Omega \subset \mathbb{R}^{2 k}$ be a bounded, smooth domain. Let $\left(N^{n}, h\right)$ be a compact, smooth Riemannian manifold without boundary, which is assumed to be isometrically embedded into some euclidean space $\mathbb{R}^{l}$. In this paper we are interested in the regularity of $k$-polyharmonic maps, which are critical points

$$
u \in W^{k, 2}(\Omega, N):=\left\{v \in W^{k, 2}\left(\Omega, \mathbb{R}^{l}\right): v(x) \in N \text { a.e. } x \in \Omega\right\}
$$

of the $k$-th order polyenergy functional:

$$
E_{k}(u)=\int_{\Omega}\left|\nabla^{k} u\right|^{2} d x
$$

Note that the functional $E_{1}$ is the Dirichlet energy for maps in $W^{1,2}(\Omega, N)$ whose critical points are harmonic maps, and $E_{2}$ is the (extrinsic) Hessian energy for maps in $W^{2,2}(\Omega, N)$ whose critical points are extrinsically biharmonic maps. Regularity issues for harmonic maps have been relatively well studied. In dimension two, the celebrated theorem by Hélein [4] asserts the interior smoothness of harmonic maps, and Qing [8] proved the boundary smoothness of harmonic maps for any smooth Dirichlet boundary data. For $k=2$ the equation of biharmonic maps is a fourth order elliptic system with borderline nonlinearities, which presents challenging problems to study their regularities. The interior regularity of extrinsic biharmonic maps to spheres

The second author is partially supported by NSF grant 0601162 . 
$N=S^{l-1} \subseteq \mathbb{R}^{l}$ has been obtained by Chang, Wang \& Yang [2]; and for general target manifolds $N \subseteq \mathbb{R}^{l}$, the second author [9] has proved the interior regularity for both extrinsic and intrinsic biharmonic maps (see Lamm \& Rivière [6] for a new proof).

Besides the $k$-th order polyenergy functional $E_{k}$, one can also consider the $k$-th order (intrinsic) polyenergy functional $F_{k}$ on $W^{k, 2}(\Omega, N)$ which is defined by

$$
F_{k}(u)=\int_{\Omega}\left|D^{k-1} \nabla u\right|^{2},
$$

where $D$ denotes the covariant derivative of the pull-back bundle $u^{\star} T N$. A critical point $u \in W^{k, 2}(\Omega, N)$ of $F_{k}$ is called an intrinsic $k$-polyharmonic map. It is well known that $F_{2}(u)=\int_{\Omega}|\tau(u)|^{2}$ is the $L^{2}$-integral of the tension field of $u$. Hence any harmonic map $u \in W^{2,2}(\Omega, N)$ satisfies $F_{2}(u)=0$ and is a global minimum of $F_{2}$.

Very recently, there have been works on the interior regularity of both extrinsic and intrinsic $k$-polyharmonic maps in the critical dimension by Gastel \& Scheven [3] for $k \geq 3$. See also Angelsberg \& Pumberger [1] for further results on $k$-polyharmonic maps.

In this paper we consider the Dirichlet boundary value problem for both extrinsic and intrinsic $k$-polyharmonic maps $u \in W^{k, 2}(\Omega, N)$. To state the boundary condition precisely, denote $\bar{\Omega}=\Omega \cup \partial \Omega$ and for $\delta>0$, define

$$
\Omega_{\delta}=\{x \in \bar{\Omega}: \operatorname{dist}(x, \partial \Omega) \leq \delta\} .
$$

Assume that for a small $\delta>0, \Phi \in C^{\infty}\left(\Omega_{\delta}, N\right)$ is a given map. Then we say $u \in W^{k, 2}(\Omega, N)$ has $\Phi$ as its Dirichlet boundary value, if

$$
\nabla^{\alpha} u=\nabla^{\alpha} \Phi \text { on } \partial \Omega
$$

holds in the sense of traces for all $2 k$-dimensional multi-indices $\alpha$ with $|\alpha| \leq k-1$.

We would like to point out that a general boundary condition such as

$$
\frac{\partial^{m} u}{\partial \nu^{m}}=\Phi_{m} \text { on } \partial \Omega, \quad 0 \leq m \leq k-1
$$

can be reduced to (1.3).

We now state our main theorem.

Theorem 1.1. For $k \in \mathbb{N}$ let $\Omega \subset \mathbb{R}^{2 k}$ be a smooth, bounded domain and $\left(N^{n}, h\right) \subseteq$ $\mathbb{R}^{l}$ be a compact, smooth Riemannian manifold without boundary. Moreover let $\Phi \in$ $C^{\infty}\left(\Omega_{\delta}, N\right)$ be given for some small $\delta>0$. Suppose that $u \in W^{k, 2}(\Omega, N)$ is an extrinsic (or intrinsic) $k$-polyharmonic map that satisfies the boundary condition (1.3). Then $u \in C^{\infty}\left(\bar{\Omega}_{\delta}, N\right)$.

We remark that for $k=2$ and $N=S^{l-1} \subseteq \mathbb{R}^{l}$, the above boundary regularity has been proved by $\mathrm{Ku}[5]$. 
The first step to prove Theorem 1.1 is the boundary Hölder regularity, which is based on the interior regularity estimates (see [4] for $k=1$, [2, 9] for $k=2$, [3] for $k \geq 3$ ), and a boundary maximum principle for $k$-polyharmonic maps with small $k$-polyenergies. An immediate consequence of the maximum principle is an $L^{\infty}$ estimate of $u-\Phi$, which yields that $u$ is continuous up to the boundary. By choosing $u-\Phi$ as a test function to (2.4) and utilizing the growth condition (2.5), we then show that $u$ is Hölder continuous near the boundary. Once we have Hölder continuity of $u$, we can modify the induction argument of Gastel \& Scheven [3] to obtain Hölder continuity of $\nabla^{k} u$ near the boundary. Finally, the smoothness of $u$ near the boundary follows from the classical Schauder theory for linear uniformly elliptic equations.

The rest of the paper is written as follows. In $\S 2$, we present some preliminary results that are needed later on. In $\S 3$, we show the boundary maximum principle under the small energy condition. In $\$ 4$, we prove the boundary Hölder continuity. In $\S 5$, we sketch the higher order regularity near the boundary.

\section{Preliminaries}

In this section, we first recall the Euler-Langrange equation for $k$-polyharmonic maps derived in [3], the interior regularity for $k$-polyharmonic maps due to [4, 9, 3], and then prove a Courant-Lebesgue type lemma for $W^{k, 2}$-maps.

If $\Pi(y): \mathbb{R}^{l} \rightarrow T_{y} N$ denotes the orthogonal projection map for $y \in N$, then a direct calculation implies that any extrinsic $k$-polyharmonic map $u \in W^{k, 2}(\Omega, N)$ satisfies

$$
\Delta^{k} u \perp T_{u} N
$$

in the weak sense, or equivalently,

$$
\int_{\Omega}\left\langle\nabla^{k} u, \nabla^{k}(\Pi(u)(V))\right\rangle=0, \quad \forall V \in C_{0}^{\infty}\left(\Omega, \mathbb{R}^{l}\right) .
$$

As in $\$ 4$ of [3], we can apply the product rule inductively to show that (2.2) is equivalent to

$$
\begin{aligned}
\int_{\Omega}\left\langle\nabla^{k} u, \nabla^{k} V\right\rangle= & \sum_{m=0}^{k-2}\left(\begin{array}{c}
k-1 \\
m
\end{array}\right) \int_{\Omega}\left\langle\nabla^{k-1-m}(\Pi(u)) \nabla^{m+1} u, \nabla^{k} V\right\rangle \\
& -\sum_{m=0}^{k-1}\left(\begin{array}{l}
k \\
m
\end{array}\right) \int_{\Omega}\left\langle\nabla^{k} u, \nabla^{k-m}(\Pi(u)) \nabla^{m} V\right\rangle .
\end{aligned}
$$

The Euler-Lagrange equation for intrinsic $k$-polyharmonic maps, similar to (2.3), has also been derived by [3], §8. It is not hard to see that (2.3) yields the following lemma. 
Lemma 2.1. Let $u \in W^{k, 2}(\Omega, N)$ be an extrinsic (or intrinsic) $k$-polyharmonic map. Then $u$ satisfies, in the sense of distribution,

$$
\Delta^{k} u=\sum_{i=0}^{k-1} \operatorname{div}^{i} g_{i}
$$

where the term $\left(g_{0}, \ldots, g_{k-1}\right)$ satisfies the growth condition:

$$
\left|g_{i}\right| \leq C \sum_{l=1}^{k}\left|\nabla^{l} u\right|^{\frac{2 k-i}{l}}, \quad i=0, \ldots, k-1,
$$

for some $C=C(k, N)>0$, where $\operatorname{div}^{i}$ denotes the $i$-th divergence operator that is inductively defined by $\operatorname{div}^{1}=\operatorname{div}$ and $\operatorname{div}^{i}=\operatorname{div}\left(\operatorname{div}^{i-1}\right)$ for $i \geq 2$.

We now introduce some further notations. For $x \in \bar{\Omega}$, define

$$
B_{R}(x)=\left\{y \in \mathbb{R}^{2 k}:|y-x| \leq R\right\}, \quad \Omega_{R}(x)=\Omega \cap B_{R}(x),
$$

and

$$
\begin{gathered}
B_{R}^{+}=\left\{y=\left(y^{\prime}, y_{2 k}\right) \in B_{R}(0) \mid y_{2 k} \geq 0\right\}, \quad T_{R}=\partial B_{R}^{+} \cap\left\{y \in \mathbb{R}^{2 k} \mid y_{2 k}=0\right\}, \\
E(u, G)=\sum_{i=1}^{k}\left(\int_{G}\left|\nabla^{i} u\right|^{\frac{2 k}{i}}\right)^{\frac{i}{k}}, \quad G \subseteq \Omega .
\end{gathered}
$$

Now we recall the interior regularity for $k$-polyharmonic maps in $\mathbb{R}^{2 k}$.

Theorem 2.2. For $k \in \mathbb{N}$ and $\Omega \subseteq \mathbb{R}^{2 k}$, there exist $\varepsilon_{0}>0$ and $\alpha_{0} \in(0,1)$ such that if $u \in W^{k, 2}(\Omega, N)$ is an extrinsic (or intrinsic) $k$-polyharmonic map satisfying, for some $x_{0} \in \Omega$ and $0<r_{0}<\frac{1}{2} \operatorname{dist}\left(x_{0}, \partial \Omega\right)$,

$$
E\left(u, B_{2 r_{0}}\left(x_{0}\right)\right) \leq \varepsilon_{0}^{2},
$$

then $u \in C^{\alpha_{0}}\left(B_{r_{0}}\left(x_{0}\right), N\right)$ and

$$
\operatorname{osc}_{B_{\tau}\left(x_{0}\right)} u \leq C\left(\frac{\tau}{r_{0}}\right)^{\alpha_{0}}\left(E\left(u, B_{2 r_{0}}\left(x_{0}\right)\right)\right)^{\frac{1}{2}}, \quad 0<\tau \leq r_{0} .
$$

Furthermore $u \in C^{\infty}(\Omega, N)$.

Proof. The reader can refer to [4] for $k=1,[2,9,6]$ for $k=2$, and [3] for $k \geq 3$. Note that by the absolute continuity, the condition (2.7) holds at any $x \in \Omega$ provided $r_{0}>0$ is chosen to be sufficiently small. 
The next lemma is a version of the Courant-Lebesgue lemma.

Lemma 2.3. Let $k \in \mathbb{N}, x_{0} \in \Omega$ and $r_{0}=\operatorname{dist}\left(x_{0}, \partial \Omega\right)>0$. For any map $u \in$ $W^{k, 2}\left(\Omega_{2 r_{0}}\left(x_{0}\right), N\right)$ there exists $r_{1} \in\left(r_{0}, 2 r_{0}\right)$ such that

$$
\operatorname{osc}_{\partial B_{r_{1}}\left(x_{0}\right) \cap \Omega} u \leq C E\left(u, \Omega_{2 r_{0}}\left(x_{0}\right)\right)^{\frac{1}{2}} .
$$

Proof. For $x \in B_{2 r_{0}}\left(x_{0}\right)$, set $r=\left|x-x_{0}\right| \in\left[0,2 r_{0}\right]$. By Fubini's theorem, we have

$$
\begin{aligned}
\int_{\Omega_{2 r_{0}}\left(x_{0}\right)}|\nabla u|^{2 k} & \geq \int_{r_{0}}^{2 r_{0}}\left(r \int_{\partial B_{r}\left(x_{0}\right) \cap \Omega}\left|\nabla_{T} u\right|^{2 k} d H^{2 k-1}\right) \frac{1}{r} d r \\
& \geq \inf _{r_{0} \leq r \leq 2 r_{0}}\left(r \int_{\partial B_{r}\left(x_{0}\right) \cap \Omega}\left|\nabla_{T} u\right|^{2 k} d H^{2 k-1}\right) \cdot\left(\int_{r_{0}}^{2 r_{0}} \frac{d r}{r}\right) \\
& \geq \ln 2 \inf _{r_{0} \leq r \leq 2 r_{0}}\left(r \int_{\partial B_{r}\left(x_{0}\right) \cap \Omega}\left|\nabla_{T} u\right|^{2 k} d H^{2 k-1}\right),
\end{aligned}
$$

where $\nabla_{T}$ denotes the gradient operator on $\partial B_{r}\left(x_{0}\right)$ and $d H^{2 k-1}$ is the area element on $\partial B_{r}\left(x_{0}\right)$. Therefore there exists $r_{1} \in\left(r_{0}, 2 r_{0}\right)$ such that

$$
r_{1} \int_{\partial B_{r_{1}}\left(x_{0}\right) \cap \Omega}\left|\nabla_{T} u\right|^{2 k} d H^{2 k-1} \leq \frac{1}{\ln 2} \int_{\Omega_{2 r_{0}}\left(x_{0}\right)}|\nabla u|^{2 k}
$$

Hence $u\left(r_{1}, \cdot\right) \in W^{1,2 k}\left(\partial B_{r_{1}}\left(x_{0}\right) \cap \Omega, N\right)$ and the Sobolev embedding theorem implies that $u\left(r_{1}, \cdot\right) \in C^{\frac{1}{2 k}}\left(\partial B_{r_{1}}\left(x_{0}\right) \cap \Omega, N\right)$, and

$$
\operatorname{osc}_{\partial B_{r_{1}}\left(x_{0}\right) \cap \Omega} u \leq C\left(r_{1} \int_{\partial B_{r_{1}}\left(x_{0}\right) \cap \Omega}\left|\nabla_{T} u\right|^{2 k} d H^{2 k-1}\right)^{\frac{1}{2 k}} \leq C E\left(u, \Omega_{2 r_{0}}\left(x_{0}\right)\right)^{\frac{1}{2}} .
$$

This completes the proof of the lemma.

We will also need the following version of the Sobolev-Poincaré inequality.

Lemma 2.4. For $k \in \mathbb{N}$, let $u \in W^{k, 2}\left(B_{2}^{+}, \mathbb{R}^{l}\right)$ with $\nabla^{\alpha} u=0$ on $T_{2}$ for all $2 k$ dimensional multiindices $\alpha$ satisfying $|\alpha| \leq k-1$. Then for all $0<r \leq 1$ and all $0 \leq i \leq k-1$, it holds

$$
\begin{aligned}
\int_{B_{2 r}^{+} \backslash B_{r}^{+}}\left|\nabla^{i} u\right|^{2} & \leq C r^{2(k-i)} \int_{B_{2 r}^{+} \backslash B_{r}^{+}}\left|\nabla^{k} u\right|^{2}, \\
E\left(u, B_{r}^{+}\right) & \leq C \int_{B_{r}^{+}}\left|\nabla^{k} u\right|^{2} .
\end{aligned}
$$


Proof. We argue by contradiction. Suppose (2.9) were false for some $0 \leq i_{0} \leq k-1$. Then there exist $\left\{u_{m}\right\} \in W^{k, 2}\left(B_{2}^{+}, \mathbb{R}^{l}\right)$ such that

$$
\int_{B_{2 r}^{+} \backslash B_{r}^{+}}\left|\nabla^{i_{0}} u_{m}\right|^{2} \geq m r^{2\left(k-i_{0}\right)} \int_{B_{2 r}^{+} \backslash B_{r}^{+}}\left|\nabla^{k} u_{m}\right|^{2}
$$

and $\nabla^{\alpha} u_{m}=0$ on $T_{2}$ for all $|\alpha| \leq k-1$. By a scaling argument we may assume that $r=1$ and

$$
\int_{B_{2}^{+} \backslash B_{1}^{+}}\left|\nabla^{i_{0}} u_{m}\right|^{2}=1 \text {. }
$$

Therefore $\left\{u_{m}\right\} \in W^{k, 2}\left(B_{2}^{+} \backslash B_{1}^{+}\right)$is bounded and we may assume that $u_{m} \rightarrow u_{\infty}$ weakly in $W^{k, 2}\left(B_{2}^{+} \backslash B_{1}^{+}\right)$and strongly in $W^{i_{0}, 2}\left(B_{2}^{+} \backslash B_{1}^{+}\right)$. By the lower semicontinuity, we have that $\nabla^{k} u_{\infty}=0$ a.e. $B_{2}^{+} \backslash B_{1}^{+}$. This, combined with $\nabla^{\alpha} u_{\infty}=0$ on $T_{2} \backslash T_{1}$ for $|\alpha| \leq k-1$, implies $u_{\infty}=0$ on $B_{2}^{+} \backslash B_{1}^{+}$. On the other hand, we have

$$
\int_{B_{2}^{+} \backslash B_{1}^{+}}\left|\nabla^{i_{0}} u_{\infty}\right|^{2}=1 \text {. }
$$

We get a desired contradiction. (2.10) is a consequence of (2.9) and the Sobolev embedding.

\section{Maximum principle}

In this section, we derive a boundary maximum principle for $k$-polyharmonic maps under a smallness condition on $E$. A similar result for harmonic maps in dimension two was obtained by Qing [8] (see [7] for $n$-harmonic maps in dimension $n$ ).

Theorem 3.1. For $k \in \mathbb{N}$ and $\Phi \in C^{\infty}\left(\Omega_{\delta}, N\right)$ for some $\delta>0$, there exists $\varepsilon_{0}>0$ such that if $u \in W^{k, 2}(\Omega, N)$ is an extrinsic (or intrinsic) $k$-polyharmonic map which satisfies the boundary condition (1.3) and for some $x \in \bar{\Omega}$ and some $R>0$,

$$
E\left(u, \Omega_{R}(x)\right) \leq \varepsilon_{0}^{2}
$$

then for any $q \in \mathbb{R}^{l}$ it holds

$$
\max _{\Omega_{R}(x)}|u-q| \leq C\left(\max _{\partial \Omega_{R}(x)}|u-q|+E\left(u, \Omega_{R}(x)\right)^{\frac{1}{2}}\right) .
$$

Proof. Let $q \in \mathbb{R}^{l}$ be fixed, denote $M=\max _{\Omega_{R}(x)}|u-q|$. We may assume that

$$
M \geq E\left(u, \Omega_{R}(x)\right)^{\frac{1}{2}} .
$$

Choose $x_{0} \in \Omega_{R}(x)$ such that

$$
\left|u\left(x_{0}\right)-q\right| \geq \frac{3}{4} M .
$$


Denote $r_{0}=\operatorname{dist}\left(x_{0}, \partial \Omega_{R}(x)\right)(>0)$. By choosing sufficiently small $\varepsilon_{0}>0$, Theorem 2.2 implies that there is $\alpha_{0} \in(0,1)$ such that

$$
\operatorname{osc}_{B_{r}\left(x_{0}\right)} u \leq C\left(\frac{r}{r_{0}}\right)^{\alpha_{0}} E\left(u, \Omega_{R}(x)\right)^{\frac{1}{2}} \leq C M\left(\frac{r}{r_{0}}\right)^{\alpha_{0}}, \quad 0<r \leq \frac{r_{0}}{2} .
$$

Hence for $r_{1}=\frac{r_{0}}{(4 C)^{\frac{1}{\alpha_{0}}}}$, we have

$$
\operatorname{osc}_{B_{r_{1}}\left(x_{0}\right)} u \leq \frac{1}{4} M
$$

This, combined with (3.3), yields

$$
\inf _{B_{r_{1}}\left(x_{0}\right)}|u-q| \geq \frac{1}{2} M .
$$

By Lemma 2.3, there is $r_{2} \in\left(r_{0}, 2 r_{0}\right)$ such that

$$
\operatorname{osc}_{\partial B_{r_{2}}\left(x_{0}\right) \cap \Omega_{R}(x)} u \leq C E\left(u, \Omega_{R}(x)\right)^{\frac{1}{2}} \text {. }
$$

Note that $\partial B_{r_{2}}\left(x_{0}\right) \cap \partial \Omega_{R}(x) \neq \varnothing$. Let $(r, \theta)$ be the polar coordinates centered at $x_{0}$. Then we can estimate

$$
\begin{aligned}
& \inf _{\substack{\left(r_{1}, \theta\right) \in\left\{r_{1}\right\} \times S^{2 k-1} \cap \Omega_{R}(x) \\
\left(r_{2}, \theta\right) \in\left\{r_{2}\right\} \times S^{2 k-1} \cap \Omega_{R}(x)}}\left|u\left(r_{1}, \theta\right)-u\left(r_{2}, \theta\right)\right| \\
& \leq C \int_{S^{2 k-1}} \int_{r_{1}}^{r_{2}}\left|u_{r}\right| \chi_{\left(\left[r_{1}, r_{2}\right] \times S^{2 k-1}\right) \cap \Omega_{R}(x)}(r, \theta) d r d \theta \\
& \leq \frac{C}{r_{1}^{2 k-1}} \int_{B_{2 r_{0}} \cap \Omega_{R}(x)}\left|u_{r}\right| d x \\
& \leq C \frac{r_{0}^{2 k-1}}{r_{1}^{2 k-1}}\left(\int_{\Omega_{R}(x)}|\nabla u|^{2 k}\right)^{\frac{1}{2 k}} \\
& \leq C E\left(u, \Omega_{R}(x)\right)^{\frac{1}{2}} .
\end{aligned}
$$

Using (3.4), (3.5), (3.6), (3.7) and choosing $x^{\star} \in \partial B_{r_{2}}\left(x_{0}\right) \cap \partial \Omega_{R}(x)$, we have

$$
\begin{aligned}
\frac{1}{2} M & \leq \inf _{B_{r_{1}}\left(x_{0}\right)}|u-q| \\
\leq & \inf _{S^{2 k-1}}\left|u\left(r_{1}, \theta\right)-u\left(r_{2}, \theta\right)\right| \\
& \quad+\sup _{\left(r_{2}, \theta\right) \in \partial B_{r_{2}}\left(x_{0}\right) \cap \Omega_{R}(x)}\left|u\left(r_{2}, \theta\right)-u\left(x^{\star}\right)\right|+\left|u\left(x^{\star}\right)-q\right| \\
& \leq \inf _{S^{2 k-1}}\left|u\left(r_{1}, \theta\right)-u\left(r_{2}, \theta\right)\right|+\operatorname{osc}_{\partial B_{r_{2}}\left(x_{0}\right) \cap \Omega_{R}(x)} u+\sup _{\partial \Omega_{R}(x)}|u-q| \\
& \leq C E\left(u, \Omega_{R}(x)\right)^{\frac{1}{2}}+\sup _{\partial \Omega_{R}(x)}|u-q| .
\end{aligned}
$$

This clearly implies (3.2). The proof is complete. 


\section{Hölder continuity near the boundary}

In this section, we will establish the Hölder continuity of $k$-polyharmonic maps near $\partial \Omega$.

In a first step we consider the case where $0 \in \partial \Omega$ and where $\Omega_{1}(0)=B_{1}^{+}$. At the end of this section we will then discuss the changes which are necessary for handling the general case. For $0<r \leq \frac{1}{2}$, let $\eta \in C^{\infty}\left(B_{1}^{+}\right)$be such that $0 \leq \eta \leq 1$ and

$$
\eta \equiv 1 \text { on } B_{r}^{+}, \quad \eta \equiv 0 \text { on } B_{1}^{+} \backslash B_{2 r}^{+} \text {and }\left\|\nabla^{i} \eta\right\|_{L^{\infty}} \leq \frac{C}{r^{i}}, \quad 1 \leq i \leq 2 k \text {. }
$$

We need a modified version of the estimate (2.10).

Lemma 4.1. For $k \in \mathbb{N}, \Phi \in C^{\infty}\left(\overline{B_{1}^{+}}, N\right)$, if $u \in W^{k, 2}\left(B_{1}^{+}, N\right)$ satisfies $\nabla^{\alpha} u=$ $\nabla^{\alpha} \Phi$ on $T_{1}$ for all $2 k$-dimensional multi-indices $\alpha$ with $|\alpha| \leq k-1$, then for all $1 \leq m \leq k-1$, we have

$$
\begin{aligned}
\int_{B_{1}^{+}} \eta^{2 k}\left|\nabla^{m} u\right|^{\frac{2 k}{m}} \leq C_{1} & \left(E\left(u, B_{1}^{+}\right)\right)^{\frac{k-m}{m}} \int_{B_{1}^{+}} \eta^{2 k}\left|\nabla^{k} u\right|^{2} \\
& +C_{2}\left[\left(\int_{B_{2 r}^{+} \backslash B_{r}^{+}}\left|\nabla^{k} u\right|^{2}\right)^{\frac{k}{m}}+\|\Phi\|_{C^{k}\left(B_{1}^{+}\right)}^{\frac{2 k}{m}} r^{2 k}\right]
\end{aligned}
$$

for some $C_{1}, C_{2}>0$ depending only only $k$, where $\eta$ is given by (4.1).

Proof. Applying (2.9) with $u-\Phi$ and the Hölder inequality, we get

$$
\begin{gathered}
\left(\int_{B_{1}^{+}} \eta^{2 k}\left|\nabla^{m}(u-\Phi)\right|^{\frac{2 k}{m}}\right)^{\frac{m}{k}} \\
\leq C \sum_{i=0}^{k-m} \int_{B_{1}^{+}}\left|\nabla^{i}\left(\eta^{m}\right)\right|^{2}\left|\nabla^{k-i}(u-\Phi)\right|^{2} \\
\leq C \int_{B_{1}^{+}} \eta^{2 m}\left|\nabla^{k} u\right|^{2}+C\left(\int_{B_{2 r}^{+} \backslash B_{r}^{+}}\left|\nabla^{k} u\right|^{2}+r^{2 k}\|\Phi\|_{C^{k}\left(B_{1}^{+}\right)}^{2}\right) \\
\leq C\left(\int_{B_{2 r}^{+}}\left|\nabla^{k} u\right|^{2}\right)^{\frac{k-m}{k}}\left(\int_{B_{1}^{+}} \eta^{2 k}\left|\nabla^{k} u\right|^{2}\right)^{\frac{m}{k}} \\
\quad+C\left(\int_{B_{2 r}^{+} \backslash B_{r}^{+}}\left|\nabla^{k} u\right|^{2}+\|\Phi\|_{C^{k}\left(B_{1}^{+}\right)}^{r^{2 k}}\right) .
\end{gathered}
$$

It is easy to see that (4.2) follows from this inequality.

Now we prove the energy decay estimate at the boundary, under a smallness condition on $E$. 
Lemma 4.2. For $k \in \mathbb{N}$ and $\Phi \in C^{\infty}\left(\overline{B_{1}^{+}}, N\right)$, there exist $\varepsilon_{0}>0,0<r_{0} \leq 1$, $\theta_{0} \in(0,1)$, and $C_{0}>0$ such that if $u \in W^{k, 2}\left(B_{1}^{+}, N\right)$ is a k-polyharmonic map satisfying (1.3) on $T_{1}$ and if

$$
E\left(u, B_{1}^{+}\right) \leq \varepsilon_{0}^{2}
$$

then

$$
E\left(u, B_{r}^{+}\right) \leq \theta_{0} E\left(u, B_{2 r}^{+}\right)+C_{0} r^{2}, \quad \forall 0<r \leq \frac{r_{0}}{2} .
$$

Proof. Let $\varepsilon_{0}>0$ be the minimum of the constants given by (2.7) and (3.1). By the Courant-Lebesgue Lemma we get $r_{0} \in\left(\varepsilon_{0}, 2 \varepsilon_{0}\right)$ such that

$$
\operatorname{osc}_{\partial B_{r_{0}} \cap \mathbb{R}_{+}^{2 k}} u \leq C E\left(u, B_{1}^{+}\right)^{\frac{1}{2}} \leq C \epsilon_{0} .
$$

Then we have

$$
\sup _{\partial B_{r_{0}}^{+}}|u-\Phi(0)| \leq C\left(\|\nabla \Phi\|_{C^{0}\left(B_{1}^{+}\right)} r_{0}+\varepsilon_{0}\right) \leq C \varepsilon_{0} .
$$

Therefore Theorem 3.1 implies that

$$
\sup _{B_{r_{0}}^{+}}|u-\Phi(0)| \leq C \varepsilon_{0} .
$$

In particular we have

$$
\|u-\Phi\|_{L^{\infty}\left(B_{r_{0}}^{+}\right)} \leq C \varepsilon_{0} .
$$

To show that $u$ is Hölder continuous we employ a hole filling argument similar to [3]. More precisely, for any $r<\frac{r_{0}}{2}$, let $\eta \in C^{\infty}\left(B_{1}^{+}\right)$be given by (4.1). Multiplying (2.4) by $\eta^{2 k}(u-\Phi)$ and integrating over $B_{1}^{+}$, we have

$$
\begin{aligned}
& \int_{B_{1}^{+}} \eta^{2 k}\left|\nabla^{k} u\right|^{2} \leq \int_{B_{1}^{+}} \eta^{2 k}\left\langle\nabla^{k} u, \nabla^{k} \Phi\right\rangle \\
&+C \sum_{p=1}^{k} \int_{B_{1}^{+}}\left|\nabla^{p}\left(\eta^{2 k}\right)\right|\left|\nabla^{k} u\right|\left|\nabla^{k-p}(u-\Phi)\right| \\
&+\int_{B_{1}^{+}} \eta^{2 k}\left|g_{0}\right||u-\Phi|+\sum_{m=1}^{k-1} \int_{B_{1}^{+}}\left|g_{m}\right|\left|\nabla^{m}\left(\eta^{2 k}(u-\Phi)\right)\right| \\
&=I+\cdots+I V .
\end{aligned}
$$

We estimate $I, \ldots, I V$ separately. For any $\delta>0$, Young's inequality implies

$$
|I| \leq \delta \int_{B_{1}^{+}} \eta^{2 k}\left|\nabla^{k} u\right|^{2}+C_{\delta}\|\Phi\|_{C^{k}\left(B_{1}^{+}\right)}^{2} r^{2 k} .
$$


Applying Young's inequality and (2.9) to $u-\Phi$ we have

$$
\begin{aligned}
|I I| & \leq \delta \int_{B_{1}^{+}} \eta^{2 k}\left|\nabla^{k} u\right|^{2}+C_{\delta} \sum_{p=1}^{k} r^{-2 p} \int_{B_{2 r}^{+} \backslash B_{r}^{+}}\left|\nabla^{k-p}(u-\Phi)\right|^{2} \\
& \leq \delta \int_{B_{1}^{+}} \eta^{2 k}\left|\nabla^{k} u\right|^{2}+C_{\delta} \int_{B_{2 r}^{+} \backslash B_{r}^{+}}\left|\nabla^{k} u\right|^{2}+C\left(\|\Phi\|_{C^{k}\left(B_{1}^{+}\right)}, \delta\right) r^{2 k} .
\end{aligned}
$$

For III we use (2.5), (4.5) and Lemma 4.1 to get

$$
\begin{aligned}
|I I I| & \leq C \varepsilon_{0} \sum_{p=1}^{k} \int_{B_{1}^{+}} \eta^{2 k}\left|\nabla^{p} u\right|^{\frac{2 k}{p}} \\
& \leq C\left(\varepsilon_{0} \int_{B_{1}^{+}} \eta^{2 k}\left|\nabla^{k} u\right|^{2}+\varepsilon_{0}^{\frac{2}{k-1}} \int_{B_{2 r}^{+} \backslash B_{r}^{+}}\left|\nabla^{k} u\right|^{2}+r^{2 k}\right) .
\end{aligned}
$$

Applying Young's inequality with $p=\frac{2 k}{2 k-m}$ and $q=\frac{2 k}{m}$ for $1 \leq m \leq k-1$, we have

$$
|I V| \leq \sum_{m=1}^{k-1}\left(\delta \int_{B_{1}^{+}} \eta^{2 k}\left|g_{m}\right|^{\frac{2 k}{2 k-m}}+C_{\delta} \int_{B_{1}^{+}}\left|\nabla^{m}\left(\eta^{m}(u-\Phi)\right)\right|^{\frac{2 k}{m}}\right) .
$$

By Lemma 4.1, (2.5), Young's inequality, the Sobolev embedding $W^{k, 2} \subseteq W^{m, \frac{2 k}{m}}$ $(1 \leq m \leq k-1)$, and (2.9), we get

$$
\begin{aligned}
|I V| \leq & \sum_{m=1}^{k-1}\left(\delta \int_{B_{1}^{+}} \eta^{2 k}\left|g_{m}\right|^{\frac{2 k}{2 k-m}}+C_{\delta} \int_{B_{1}^{+}}\left|\nabla^{m}\left(\eta^{m}(u-\Phi)\right)\right|^{\frac{2 k}{m}}\right) \\
\leq & C \delta \sum_{m=1}^{k} \int_{B_{1}^{+}} \eta^{2 k}\left|\nabla^{m} u\right|^{\frac{2 k}{m}}+C_{\delta} \sum_{m=1}^{k-1}\left(\int_{B_{1}^{+}}\left|\nabla^{k}\left(\eta^{m}(u-\Phi)\right)\right|^{2}\right)^{\frac{k}{m}} \\
\leq & C\left(\delta \int_{B_{1}^{+}} \eta^{2 k}\left|\nabla^{k} u\right|^{2}+\varepsilon_{0}^{\frac{2}{k-1}} \int_{B_{2 r}^{+} \backslash B_{r}^{+}}\left|\nabla^{k} u\right|^{2}+r^{2 k}\right) \\
& +C_{\delta} \sum_{m=1}^{k-1}\left(\int_{B_{2 r}^{+} \backslash B_{r}^{+}}\left|\nabla^{k}(u-\Phi)\right|^{2}+\int_{B_{1}^{+}} \eta^{2 m}\left|\nabla^{k}(u-\Phi)\right|^{2}\right)^{\frac{k}{m}} .
\end{aligned}
$$

Putting together (4.6)-(4.9), we get

$$
\int_{B_{1}^{+}} \eta^{2 k}\left|\nabla^{k} u\right|^{2} \leq C_{0}\left(\delta+\varepsilon_{0}\right) \int_{B_{1}^{+}} \eta^{2 k}\left|\nabla^{k} u\right|^{2}+C\left(\int_{B_{2 r}^{+} \backslash B_{r}^{+}}\left|\nabla^{k} u\right|^{2}+r^{2 k}\right) \text {. }
$$


By choosing $\delta$ and $\varepsilon_{0}$ small enough, we obtain

$$
\int_{B_{r}^{+}}\left|\nabla^{k} u\right|^{2} \leq C\left(\int_{B_{2 r}^{+} \backslash B_{r}^{+}}\left|\nabla^{k} u\right|^{2}+r^{2 k}\right), \quad 0<r \leq \frac{r_{0}}{2} .
$$

Applying (2.10) to $u-\Phi$ and adding $C \int_{B_{r}^{+}}\left|\nabla^{k} u\right|^{2}$ to both sides of this inequality, we obtain

$$
E\left(u, B_{r}^{+}\right) \leq \theta_{0} E\left(u, B_{2 r}^{+}\right)+C r^{2},
$$

where $\theta_{0}=\frac{C}{C+1}$. This completes the proof of (4.4).

With the help of Lemma 4.2, we can now prove that an extrinsic (or intrinsic) $k$ polyharmonic map $u \in W^{k, 2}\left(B_{1}^{+}, N\right)$, satisfying (1.3), is Hölder continuous. In fact, by the absolute continuity and the scaling invariance of $E$ in $\mathbb{R}^{2 k}$, we have that for any $\varepsilon_{0}>0$ there is $r_{\star}>0$ such that

$$
\sup _{x \in T_{1}} E\left(u, B_{r_{\star}}(x)\right) \leq \varepsilon_{0}^{2} .
$$

Hence Lemma 4.2 implies that for any $x \in T_{1}$,

$$
E\left(u, B_{r}(x)\right) \leq \theta E\left(u, B_{2 r}(x)\right)+C r^{2}, \quad r<\frac{r_{0}}{2} .
$$

By a standard iteration argument, this implies that there exists $0<\alpha_{0}<1$ such that

$$
E\left(u, B_{r}(x)\right) \leq C r^{2 \alpha_{0}}, \quad \forall x \in T_{1} \text { and } 0<r \leq \frac{r_{0}}{2}
$$

Hence by Morrey's decay lemma and the interior regularity Theorem 2.2, we conclude that $u \in C_{\text {loc }}^{\alpha_{0}}\left(B_{1}^{+}, N\right)$.

Now we discuss the case of general curved boundaries. We let $x_{0} \in \partial \Omega$. Since $\Omega$ is assumed to be smooth, there exists $r_{0}>0$ and a $C^{2 k}$-diffeomorphism $\Phi: B_{1}^{+} \rightarrow$ $\Omega_{r_{0}}\left(x_{0}\right)$ such that $\Phi(0)=x_{0}$ and $\Phi\left(T_{1}\right)=\partial \Omega \cap B_{r_{0}}\left(x_{0}\right)$. We let $\Psi=\Phi^{-1}$ and we define the map $v: B_{1}^{+} \rightarrow N$ by $v(y)=u(\Phi(y))$ or written differently $u(x)=$ $v(\Psi(x))$. Now a standard calculation yields that

$$
E_{k}\left(u, \Omega_{r_{0}}\left(x_{0}\right)\right)=\int_{B_{1}^{+}}\left|\left(\nabla^{g}\right)^{k} v\right|_{g}^{2} d v_{g},
$$

where $g=\Phi^{\star} g_{0}$ is the pull-back metric of the euclidean metric $g_{0}, \nabla^{g}$ is the covariant derivative with respect to the metric $g$ and $d v_{g}$ is the volume element of $g$. Performing a similar calculation for the polyharmonic map equation satisfied by $u$ we see that the map $v$ solves the polyharmonic map equation for a general Riemannian metric $g$ in the domain. Since $\Phi$ is a diffeomorphism one can check that the resulting equation for $v$ is of the type (2.4) but with coefficients which come from the diffeomorphism and 
with additional, harmless lower order terms. Also, by the way we set up our boundary condition, we get that $v$ satisfies a similar boundary condition on $T_{1}$ with boundary data which are suitable smooth transformations of the original ones. Finally we want to mention that by an application of the transformation formula and Hölder's inequality we get the existence of a constant $c_{0}=c_{0}(\Phi) \geq 1$ such that

$$
\frac{1}{c_{0}} E\left(v, B_{1}^{+}\right) \leq E\left(u, \Omega_{r_{0}}\left(x_{0}\right)\right) \leq c_{0} E\left(v, B_{1}^{+}\right) .
$$

Combining all these facts it follows that the interior regularity Theorem 2.2 and Lemma 4.3 extend to the new equation. Hence we get from the above arguments that $v$ is $\alpha_{0}$ Hölder continuous and this directly gives that $u$ is $\alpha_{0}$ Hölder continuous.

\section{Higher order regularity near the boundary}

In this section, we outline the proof of higher order regularity for $k$-polyharmonic maps $u \in W^{k, 2}(\Omega, N)$ that satisfy the boundary condition (1.3). The idea is to show that $\nabla^{i} u$ is Hölder continuous near $\partial \Omega$ for all $1 \leq i \leq k$. The argument here is a suitable modification of [3], §7. Again we restrict our attention to the case where $0 \in \partial \Omega$ and where $\Omega_{1}(0)=B_{1}^{+}$since the general case can be reduced to this one by arguing as above.

First we need a standard estimate for $k$-polyharmonic functions satisfying homogeneous Dirichlet boundary conditions in $T_{1}$ the proof of which is standard.

Lemma 5.1. For $k \in \mathbb{N}$, let $v \in C^{\infty}\left(\overline{B_{1}^{+}}, \mathbb{R}^{l}\right)$ solve

$$
\Delta^{k} v=0 \text { in } B_{1}^{+}, \quad \nabla^{\alpha} v=0 \text { on } T_{1} \quad \forall|\alpha| \leq k-1 .
$$

Then for all $1 \leq m \leq k, r<1$, and all $0<\rho \leq \frac{r}{2}$, we have

$$
\begin{gathered}
\int_{B_{\rho}^{+}}\left|\nabla^{m} v\right|^{2} \leq C\left(\frac{\rho}{r}\right)^{2 k} \int_{B_{r}^{+}}\left|\nabla^{m} v\right|^{2}, \\
\int_{B_{\rho}^{+}}\left|\nabla^{k} v-\left(\nabla^{k} v\right)_{\rho}\right|^{2} \leq C\left(\frac{\rho}{r}\right)^{2 k+2} \int_{B_{r}^{+}}\left|\nabla^{k} v-\left(\nabla^{k} v\right)_{r}\right|^{2},
\end{gathered}
$$

where $\left(\nabla^{k} v\right)_{r}=\frac{1}{\left|B_{r}^{+}\right|} \int_{B_{r}^{+}} \nabla^{k} v$.

Now we want to show that there exists an $\varepsilon_{0}>0$ such that if

$$
E\left(u, B_{1}^{+}\right) \leq \varepsilon_{0}^{2},
$$

then for any noninteger $\gamma=[\gamma]+\beta \in(0, k), u \in C^{[\gamma], \beta}\left(B_{\frac{1}{2}}^{+}, N\right)$ and

$$
\int_{B_{r}^{+}}\left|\nabla^{k} u\right|^{2} \leq c r^{2 \gamma}, \quad \forall 0<r \leq \frac{r_{0}}{2}
$$


From (4.12), we know that this claim is true for $\gamma \in(0,1)$. Now we want to show that whenever this claim is true for some number $\gamma \in(0, k)$ then it is also true for $\gamma_{1}=\min \left(k, \frac{2 k+1}{2 k} \gamma\right)=\left[\gamma_{1}\right]+\beta_{1} \in(0, k)$. First note that by $(2.10)$ (applied to $\left.u-\Phi\right)$, (5.4) implies that for any $[\gamma]<m \leq k$,

$$
\int_{B_{r}^{+}}\left|\nabla^{m} u\right|^{\frac{2 k}{m}} \leq C r^{\frac{2 k \gamma}{m}}, \quad 0<r \leq \frac{r_{0}}{2} .
$$

This shows that for any $0 \leq m \leq k-1$ and $r \leq \frac{r_{0}}{4}$,

$$
\int_{B_{r}^{+}}\left|g_{m}\right|^{\frac{2 k}{2 k-m}} \leq C \sum_{i=1}^{k} \int_{B_{r}^{+}}\left|\nabla^{i} u\right|^{\frac{2 k}{i}} \leq C\left(r^{2 k}+\sum_{i=[\gamma]+1}^{k} r^{\frac{2 k \gamma}{i}}\right),
$$

since $\left\|\nabla^{i} u\right\|_{L^{\infty}} \leq C$ for $1 \leq i \leq[\gamma]$. Therefore we have

$$
\left(\int_{B_{r}^{+}}\left|g_{m}\right|^{\frac{2 k}{2 k-m}}\right)^{\frac{2 k-m}{2 k}} \leq C\left(r^{k+1}+r^{\frac{k+1}{k} \gamma}\right) .
$$

Let $v$ be a $k$-polyharmonic function on $B_{r}^{+}$such that $\nabla^{\alpha} v=\nabla^{\alpha}(u-\Phi)$ on $\partial B_{r}^{+}$for all $2 k$-dimensional multi-indices $\alpha$ with $|\alpha| \leq k-1$. Define $w=u-\Phi-v$. Then we have $w \in W_{0}^{k, 2}\left(B_{r}^{+}, N\right)$, and

$$
\int_{B_{r}^{+}}\left\langle\nabla^{k} v, \nabla^{k} w\right\rangle=0
$$

Note also that

$$
\int_{B_{r}^{+}} \nabla^{k} w=0
$$

Moreover by the mean value theorem we know that for any $x=\left(x^{\prime}, x^{2 k}\right) \in B_{r}^{+}$with $x^{2 k}>0$ there exist $0 \leq x_{[\gamma]}^{2 k} \leq \cdots \leq x_{1}^{2 k} \leq x^{2 k}$ such that

$$
w(x)=\nabla_{2 k} w\left(x^{\prime}, x_{1}^{2 k}\right) x^{2 k}=\cdots=\left(\nabla_{2 k}\right)^{[\gamma]} w\left(x^{\prime}, x_{[\gamma]}^{2 k}\right) x^{2 k} \cdot x_{1}^{2 k} \cdot \ldots \cdot x_{[\gamma]-1}^{2 k} .
$$

Hence we get

$$
\begin{aligned}
\|w\|_{L^{\infty}\left(B_{r}^{+}\right)} & \leq c r^{[\gamma]}\left\|\nabla^{[\gamma]}(u-\Phi-v)\right\|_{L^{\infty}\left(B_{r}^{+}\right)} \\
& \leq c r^{\gamma}\left(\|u\|_{C^{[\gamma], \beta}\left(B_{r}^{+}\right)}+\|\Phi\|_{C^{[\gamma], \beta}\left(B_{r}^{+}\right)}+\|v\|_{C^{[\gamma], \beta}\left(B_{r}^{+}\right)}\right) \\
& \leq C r^{\gamma} .
\end{aligned}
$$


Therefore, multiplying (2.4) by w, using Lemma 2.1, Young's inequality, Poincaré's inequality and the estimates (5.5), (5.6), we have

$$
\begin{aligned}
\int_{B_{r}^{+}}\left|\nabla^{k} w\right|^{2}= & \int_{B_{r}^{+}}\left\langle\nabla^{k} u, \nabla^{k} w\right\rangle-\int_{B_{r}^{+}}\left\langle\nabla^{k} \Phi-\left(\nabla^{k} \Phi\right)_{r}, \nabla^{k} w\right\rangle \\
\leq & \frac{1}{2} \int_{B_{r}^{+}}\left|\nabla^{k} w\right|^{2}+C|| w||_{L^{\infty}\left(B_{r}^{+}\right)} \int_{B_{r}^{+}}\left|g_{0}\right| \\
& \quad+C \sum_{m=1}^{k-1}\left(\int_{B_{r}^{+}}\left|g_{m}\right|^{\frac{2 k}{2 k-m}}\right)^{\frac{2 k-m}{k}}+C \int_{B_{r}^{+}}\left|\nabla^{k} \Phi-\left(\nabla^{k} \Phi\right)_{r}\right|^{2} \\
\leq & \frac{1}{2} \int_{B_{r}^{+}}\left|\nabla^{k} w\right|^{2}+C r^{\gamma}\left(r^{k+1}+r^{\frac{k+1}{k} \gamma}\right)+C\left(r^{k+1}+r^{\frac{k+1}{k} \gamma}\right)^{2} \\
\leq & \frac{1}{2} \int_{B_{r}^{+}}\left|\nabla^{k} w\right|^{2}+C r^{2 \gamma_{1}} .
\end{aligned}
$$

Hence we obtain

$$
\int_{B_{r}^{+}}\left|\nabla^{k} w\right|^{2} \leq C r^{2 \gamma_{1}}, \quad 0<r \leq \frac{r_{0}}{4} .
$$

Since $\nabla^{\alpha} w=0$ on $T_{r}$ for all $\alpha$ with $|\alpha| \leq k-1$, (2.9) implies that for all $1 \leq m \leq k$

$$
\int_{B_{r}^{+}}\left|\nabla^{m} w\right|^{2} \leq C r^{2\left(\gamma_{1}+k-m\right)}, \quad 0<r \leq \frac{r_{0}}{4} .
$$

This, combined with (5.1), yields that for any $0<r \leq \frac{1}{2}, \rho \leq \frac{r}{2}$, and $1 \leq m \leq k$

$$
\begin{aligned}
\int_{B_{\rho}^{+}}\left|\nabla^{m}(u-\Phi)\right|^{2} & \leq 2 \int_{B_{\rho}^{+}}\left|\nabla^{m} w\right|^{2}+2 \int_{B_{\rho}^{+}}\left|\nabla^{m} v\right|^{2} \\
& \leq C r^{2\left(\gamma_{1}+k-m\right)}+2 \int_{B_{\rho}^{+}}\left|\nabla^{m} v\right|^{2} \\
& \leq C r^{2\left(\gamma_{1}+k-m\right)}+C\left(\frac{\rho}{r}\right)^{2 k} \int_{B_{r}^{+}}\left|\nabla^{m} v\right|^{2} \\
& \leq C\left(\frac{\rho}{r}\right)^{2 k} \int_{B_{r}^{+}}\left|\nabla^{m}(u-\Phi)\right|^{2}+C r^{2\left(\gamma_{1}+k-m\right)}
\end{aligned}
$$

Note that this inequality is trivially true for $\frac{r}{2} \leq \rho \leq r$. By a standard iteration argument, this implies that for $m>\gamma_{1}$ and any $0<\rho \leq \frac{1}{4}$

$$
\int_{B_{\rho}^{+}}\left|\nabla^{m} u\right|^{2} \leq C \rho^{2\left(\gamma_{1}+k-m\right)} .
$$

This, together with the interior estimate of [3], implies that $u \in C^{\left[\gamma_{1}\right], \beta_{1}}\left(B_{r}^{+}, N\right)$ and satisfies (5.4). 
Next we let $\gamma_{\star}=k+\beta, 0<\beta<\frac{1}{2}$. Then (5.4) holds for $\gamma=\frac{2 k}{2 k+1} \gamma_{\star}$. Hence (5.5) remains true for this value of $\gamma$. Suppose that $v$ and $w$ are defined as above. Then we can repeat the above argument to improve the estimate (5.7) so that

$$
\int_{B_{r}^{+}}\left|\nabla^{k} w\right|^{2} \leq C r^{2 \gamma^{\star}}, \quad 0<r \leq \frac{1}{2} .
$$

Combining (5.11) with (5.2), we obtain for any $0<r \leq \frac{1}{2}$ and $0<\rho \leq \frac{r}{2}$,

$$
\begin{aligned}
\int_{B_{\rho}^{+}}\left|\nabla^{k}(u-\Phi)-\left(\nabla^{k}(u-\Phi)\right)_{\rho}\right|^{2} & \leq C \int_{B_{\rho}^{+}}\left|\nabla^{k} v-\left(\nabla^{k} v\right)_{\rho}\right|^{2}+C r^{2 \gamma_{\star}} \\
& \leq C\left(\frac{\rho}{r}\right)^{2 k+2} \int_{B_{r}^{+}}\left|\nabla^{k} v-\left(\nabla^{k} v\right)_{r}\right|^{2}+C r^{2 \gamma_{\star}} \\
& \leq C\left(\frac{\rho}{r}\right)^{2 k+2} \int_{B_{r}^{+}}\left|\nabla^{k} u-\left(\nabla^{k} u\right)_{r}\right|^{2}+C r^{2 \gamma_{\star}}
\end{aligned}
$$

This implies

$$
\int_{B_{\rho}^{+}}\left|\nabla^{k} u-\left(\nabla^{k} u\right)_{\rho}\right|^{2} \leq C\left(\frac{\rho}{r}\right)^{2 k+2} \int_{B_{r}^{+}}\left|\nabla^{k} u-\left(\nabla^{k} u\right)_{r}\right|^{2}+C r^{2 \gamma^{\star}} .
$$

It is well known that (5.12) yields

$$
\int_{B_{\rho}^{+}}\left|\nabla^{k} u-\left(\nabla^{k} u\right)_{\rho}\right|^{2} \leq C \rho^{2 \gamma_{\star}}, \quad 0<\rho \leq \frac{1}{4} .
$$

Therefore we have that $u \in C^{k, \beta}\left(B_{r}^{+}, N\right)$. Finally the higher order regularity follows from the classical Schauder theory applied to the equation (2.4).

Acknowledgments. The authors would like to thank the referee for his/or her careful reading of the paper.

\section{References}

[1] G. Angelsberg and D. Pumberger, A regularity result for polyharmonic maps with higher integrability, preprint, 2007.

[2] S.-Y. A. Chang, L. Wang and P. Yang, A regularity theory of biharmonic maps. Comm. Pure Appl. Math. 52 (1999), pp. 1113-1137.

[3] A. Gastel and C. Scheven, Regularity of polyharmonic maps in the critical dimension, preprint, 2007.

[4] F. Hélein, Régularité des applications faiblement harmoniques entre une surface et une variété riemannienne, C. R. Acad. Sci. Paris Sér. I Math. 312 (1991), pp. 591-596. 
[5] Y. Ku, Interior and boundary regularity of intrinsic biharmonic maps to spheres, Pacific J. Math. 234 (2008), pp. 43-67.

[6] T. Lamm and T. Rivière, Conservation laws for fourth order systems in four dimensions, Comm. PDE. 33 (2008), pp. 245-262.

[7] L. Mou and P. Yang, Regularity for $n$-harmonic maps, J. Geom. Anal. 6 (1996), pp. $91-$ 112.

[8] J. Qing, Boundary regularity of weakly harmonic maps from surfaces. J. Funct. Anal. 114 (1993), pp. 458-466.

[9] C. Wang, Biharmonic maps from $\mathbb{R}^{4}$ into a Riemannian manifold. Math. Z. 247 (2004), pp. $65-87$.

Received March 13, 2008; revised June 26, 2008.

\section{Author information}

Tobias Lamm, Max-Planck-Institute for Gravitational Physics, Am Mühlenberg 1, 14476 Golm, Germany.

Current address:

Department of Mathematics, University of British Columbia, 1984 Mathematics Road, Vancouver, BC V6T 1Z2, Canada.

E-mail: tlamm@aei.mpg.de

Changyou Wang, Department of Mathematics, University of Kentucky,

Lexington, KY 40506-0027, USA.

E-mail: cywang@ms . uky . edu 